This is an author-created, un-copyedited version of an article accepted for publication/published in Modelling and Simulation in Materials Science and Engineering. IOP Publishing Ltd is not responsible for any errors or omissions in this version of the manuscript or any version derived from it. The Version of Record is available online at http://dx.doi.org/10.1088/0965-0393/24/4/045017. Citation details: Nöhring, W. G.; Curtin, W. A. Model. Simul. Mater. Sci. Eng. 2016, 24 (4), 45017.

\title{
Thermodynamic properties of average-atom interatomic potentials for alloys
}

\author{
Wolfram Georg Nöhring* and William Arthur Curtin \\ Institute of Mechanical Engineering, École Polytechnique Fédérale de Lausanne, EPFL STI IGM Station 9, CH-1015 Lausanne
}

(Dated: November 16, 2015)

\begin{abstract}
The atomistic mechanisms of deformation in multicomponent random alloys are challenging to model because of their extensive structural and compositional disorder. For embedded-atom-method interatomic potentials, a formal averaging procedure can generate an average-atom EAM potential and this average-atom potential has recently been shown to accurately predict many zero-temperature properties of the true random alloy. Here, the finite-temperature thermodynamic properties of the average-atom potential are investigated to determine if the average-atom potential can represent the true random alloy Helmholtz Free Energy as well as important finite-temperature properties. Using a thermodynamic integration approach, the average-atom system is found to have an entropy difference of at most $0.05 \mathrm{k}_{B}$ /atom relative to the true random alloy over a wide temperature range, as demonstrated on $\mathrm{FeNiCr}$ and $\mathrm{Ni}_{85} \mathrm{Al}_{15}$ model alloys. Lattice constants, and thus thermal expansion, and elastic constants are also well-predicted (within a few percent) by the average-atom potential over a wide temperature range. The largest differences between the average atom and true random alloy are found in the zero temperature properties, which reflect the role of local structural disorder in the true random alloy. Thus, the average-atom potential is a valuable strategy for modeling alloys at finite temperatures.
\end{abstract}

Keywords: Free Energy, Molecular Dynamics, Finite Temperature, Elastic Constants, Embedded Atom Method (EAM)

\section{INTRODUCTION}

Atomistic simulation is a standard tool for studying the mechanistic origins of many complex material phenomena in metals, such as plastic flow, fracture, phase transformation kinetics, and grain boundary structures and migration. The majority of studies to date have been carried out on elemental metals, specific ordered phases, and occasionally dilute solid solutions. Real engineering materials are more often multicomponent alloys with complex precipitate phases and/or non-dilute solute concentrations. Given a set of validated interatomic potentials for the desired alloy, it is possible to execute direct atomistic simulations in complex systems but it is difficult to clearly identify operative mechanisms due to the high level of microstructural complexity. This is particularly true in non-dilute solid solutions where mechanical behavior is controlled by defects (dislocations, grain boundaries, crack tips) interacting with many solutes. Because of these challenges, a common strategy is to understand the mechanisms identified in elemental metals in terms of key material properties (e.g. elastic constants, stacking fault energies, surface or interface energies, etc.) and to apply those concepts to the real engineering materials. Such a strategy neglects, however, the additional role of structural and compositional disorder present in the complex alloys. There is thus a general need to develop additional modeling approaches that help separate the effects of the additional disorder in a complex alloy from the effects of the baseline (average) material response.

In a recent paper, related to early work $[1,2]$, the concept of an "average atom", or $A$-atom, interatomic potential has been introduced and validated for the study of defects in random alloys [3]. Specifically, within the context of embeddedatom-method (EAM) potentials [4], it is possible to perform a formal averaging over all configurations of an alloy at a given

*wolfram.nohring@epfl.ch overall composition and thus to derive a new "average atom" potential that has the EAM form. Moreover, the $A$-atom potential accurately predicts many important properties of the true random alloy including defect properties such as stacking fault energies, at zero temperature. Several useful advantages of the $A$-atom potential have been identified. For instance, the $A$-atom potential can be combined with the original true atom EAM potentials to study the interaction of true atom "solutes" with defects (e.g. dislocations) in the $A$-atom material. Such interactions are essential in understanding alloy behavior but can be very difficult to compute by brute-force simulation on random alloys. Comparisons of the $A$-atom material to the true alloy also enable the identification of what properties are controlled by "average" behavior and what properties are controlled by the fluctuations. Overall, the development and application of the $A$-atom potential is thus a valuable tool with application in several different directions of computational metallurgy.

The $A$-atom potential has been tested against the true random alloy only at zero temperature. To have wide practical utility, it is important that the $A$-atom potential provide an accurate representation of the true random alloy at finite temperatures. The purpose of the present paper is to precisely examine the thermodynamic and finite-temperature properties of the $A$-atom material as compared to the true random alloy. We use two typical model materials and examine the thermal expansion, Helmholtz Free Energy, and cubic elastic constants up to $700 \mathrm{~K}$. We find excellent agreement as compared to the true random alloys, with the major differences being in the $T=0 \mathrm{~K}$ properties only. This work thus confirms the broad validity of the $A$-atom interatomic potential for modeling the homogenized properties of random alloys.

The remainder of this paper is organized as follows. In the next section, we introduce the "average atom" interatomic potential as derived from the alloy EAM potentials. In Section 3, we examine the lattice constants versus temperature by direct MD simulations, which establishes the appropriate volumes for computation of the Helmholtz Free Energy that is dis- 
This is an author-created, un-copyedited version of an article accepted for publication/published in Modelling and Simulation in Materials Science and Engineering. IOP Publishing Ltd is not responsible for any errors or omissions in this version of the manuscript or any version derived from it. The Version of Record is available online at http://dx.doi.org/10.1088/0965-0393/24/4/045017. Citation details: Nöhring,

W. G.; Curtin, W. A. Model. Simul. Mater. Sci. Eng. 2016, 24 (4), 45017.

cussed and presented in Section 4. Section 5 presents methods and results for the cubic elastic constants versus temperature. Section 6 summarizes our findings.

\section{AVERAGE EAM POTENTIALS}

Consider an alloy with $N_{T}$ distinct atom types and a total of $N$ atoms. Let $\{X, Y\}$ refer to atom types and $\{i, j\}$ to atom sites. Further, consider the set of occupation variables $\left\{s_{i}^{X}\right\}$, where $s_{i}^{X}=1$ if the atom at site $i$ has the type $X$ and zero otherwise. Within the EAM interatomic potential description, the potential energy of this system is

$$
E\left(\left\{s_{i}^{X}\right\}\right)=\sum_{i}^{N} \sum_{X}^{N_{T}} s_{i}^{X} F^{X}\left(\rho_{i}\right)+\frac{1}{2} \sum_{i, j \neq i}^{N} \sum_{X, Y}^{N_{T}} s_{i}^{X} s_{j}^{Y} V_{i j}^{X Y},
$$

where

$$
\rho_{i}=\sum_{j \neq i}^{N} \sum_{X}^{N_{T}} s_{j}^{X} \rho_{i j}^{X}
$$

$V_{i j}^{X Y}$ is the pair potential function, $F^{X}\left(\rho_{i}\right)$ the embedding energy functional, $\rho_{i}$ the total electron density at site $i$, and $\rho^{X}\left(r_{i j}\right)$ is the contribution of an $X$-atom at site $j$ to $\rho_{i}$.

A simple approximation for the configurational average of Eq. 1 can be obtained for a random solution, i.e. a solution with uncorrelated $\left\{s_{i}^{X}\right\}[1,3]$. The derivation in [3] proceeds in two steps. First, Eq. 1 is averaged over all occupations compatible with the average concentrations $c^{X}$. The assumption of uncorrelated $\left\{s_{i}^{X}\right\}$ implies $\left\langle s_{i}^{X} s_{j}^{Y}\right\rangle=c^{X} c^{Y}$, where $\langle\ldots\rangle$ indicates the average. The average energy is therefore

$$
\langle E\rangle=\sum_{i}^{N} \sum_{X}^{N_{T}} c^{X}\left\langle F^{X}\left(\rho_{i}\right)\right\rangle+\frac{1}{2} \sum_{i, j \neq i}^{N} \sum_{X, Y}^{N_{T}} c^{X} c^{Y} V_{i j}^{X Y} .
$$

Second, the embedding energy is expanded in a Taylor series around the average electron density as $\left\langle F^{X}\left(\rho_{i}\right)\right\rangle=$ $\left\langle F^{X}\left(\left\langle\rho_{i}\right\rangle\right)\right\rangle+O\left(\rho_{i}-\left\langle\rho_{i}\right\rangle\right)^{2}$. Note that averaging eliminates the first-order term of the series. By neglecting the second order term, the average energy is

$$
\langle E\rangle=\sum_{i}^{N} F^{A}\left(\left\langle\rho_{i}\right\rangle\right)+\frac{1}{2} \sum_{i, j \neq i}^{N} V_{i j}^{A A},
$$

where

$$
\begin{aligned}
F^{A}\left(\left\langle\rho_{i}\right\rangle\right) & =\sum_{X}^{N_{T}} c^{X} F^{X}\left(\left\langle\rho_{i}\right\rangle\right), \\
V_{i j}^{A A} & =\sum_{X, Y}^{N_{T}} c^{X} c^{Y} V_{i j}^{X Y}, \\
\left\langle\rho_{i}\right\rangle & =\sum_{X}^{N_{T}} c^{X} \rho_{i j}^{X},
\end{aligned}
$$

Eq. 4 is just the potential energy of an equivalent system composed of a single "average" atom type $A$, whose EAM functions are the weighted averages of the pure element functions.
Note that for molecular dynamics, one would additionally take the weighted average of the masses $m^{X}$ :

$$
m^{A}=\sum_{X}^{N_{T}} c^{X} m^{X}
$$

The $A$-atom approximation is similar to the "virtual crystal" approximation $[5,6]$. It is instructive to briefly recapitulate Wojtowicz and Kirkwood's [7] application of the virtual crystal approximation to lattice dynamics. Specifically, they model the phonon spectrum of the true disordered system as the phonon spectrum of the virtual crystal plus local perturbations in the phonon spectrum. The virtual crystal has the mean mass and force constants of the true system. In the absence of short range order, the difference between mean and true force constants generates only a second-order perturbation correction of the virtual crystal's vibrational partition function. Hence, we expect that the $A$-atom approximation describes the vibrational properties of random solutions well.

The $A$-atom potential is an entirely new interatomic potential that is independent of atomistic configuration. It is thus applicable to the study of defects in the average material, and also for studying finite-temperature properties associated with thermal vibrations. In the next sections, we will compare the $A$-atom properties at finite temperature to the true random alloy counterparts for two model materials, the equiatomic random fcc FeNiCr system using the interatomic potentials developed by Bonny et al. [8] and the $N i_{85} A l_{15}$ fcc solid solution using the interatomic potentials of Pun et al. [9]. The $\mathrm{FeNiCr}$ $A$-atom material has recently been studied at $0 \mathrm{~K}$, and is a model system for understanding "High Entropy Alloys" [10] and stainless steels. The $\mathrm{Ni}_{85} \mathrm{Al}_{15} A$-atom material is an interesting complementary system because $\mathrm{Ni}$ and $\mathrm{Al}$ have very different lattice parameters (3.52 $\AA$ vs. $4.05 \AA$ ) and elastic constants (e.g. $C_{11}: 241 \mathrm{GPa}$ vs. $114 \mathrm{GPa}$ ) at $0 \mathrm{~K}$, and the solid solution is relevant in Ni-based superalloys. All simulations here are performed using the LAMMPS package [11].

\section{LATTICE PARAMETER AND THERMAL EXPANSION}

For each alloy, we prepared three random solid solutions and one pure crystal of the corresponding average material. The samples were periodic fcc crystals, with $\langle 100\rangle$ directions parallel to the edges of the simulation cell. The sample size was $30 \times 30 \times 30$ unit cells $(108000$ atoms $)$. All samples were constructed using the lattice parameter of the $A$-atom material but relaxed by simultaneously minimizing the three normal stresses (by independently varying the cell edge lengths) and the potential energy of the atoms. After this relaxation, the potential energy was minimized at fixed cell size until the norm of the force vector fell below $10^{-6}-10^{-8} \mathrm{eV} / \AA$.

The samples were then heated to the desired temperature using a Langevin thermostat [12] with relaxation time parameter of $1 \mathrm{ps}$ and timestep of $1 \mathrm{fs}$. A Berendsen barostat [13] with relaxation time parameter of $0.01 \mathrm{ps} \times B$, where $B$ is the $\mathrm{T}=0 \mathrm{~K}$ bulk modulus, was used to maintain minimal normal 
This is an author-created, un-copyedited version of an article accepted for publication/published in Modelling and Simulation in Materials Science and Engineering. IOP Publishing Ltd is not responsible for any errors or omissions in this version of the manuscript or any version derived from it. The Version of Record is available online at http://dx.doi.org/10.1088/0965-0393/24/4/045017. Citation details: Nöhring,

W. G.; Curtin, W. A. Model. Simul. Mater. Sci. Eng. 2016, 24 (4), 45017.

stresses so that thermal expansion occurred at near-zero pressure. Due to disorder in the random solid solutions, the relaxation was slightly anisotropic even for these large simulation cell sizes. Samples were heated in increments of $50 \mathrm{~K}$. The change in temperature was introduced over $30 \mathrm{ps}$, followed by a 20 ps period of equilibration at the new temperature. The average edge lengths of the cell were then measured over a subsequent $10 \mathrm{ps}$ interval and then the three edge lengths were averaged to compute the average lattice parameter of each sample. Averages over the three random samples yield our final lattice parameter for the random alloys.

The lattice parameters for the true random alloys and the corresponding $A$-atom material are shown in Fig. 1a). The $A$-atom result agrees to within $\sim 0.0006 \AA$ for $\mathrm{FeNiCr}$ and $\sim 0.003 \AA$ for $\mathrm{Ni}_{85} \mathrm{Al}_{15}$, with most of the difference being at $T=0 \mathrm{~K}$. The $T=0 \mathrm{~K}$ difference reflects the local structural relaxations that can occur in the true random alloy. The smaller additional differences in lattice parameter at finite temperatures reflect the very small differences in anharmonicity between random and average materials, showing that the $A$-atom material gives an excellent representation of the true random alloy. The thermal expansion coefficient is the derivative of the lattice constant with respect to temperature, and is shown in Fig. 1b) for both $\mathrm{FeNiCr}$ and $\mathrm{Ni}_{85} \mathrm{Al}_{15}$ systems. In both cases, the $A$-atom material is in very good agreement with the true random alloy.

\section{FREE ENERGY}

\section{A. Methodology}

With the lattice constants established in the previous section, we can now compute the Helmholtz Free Energy difference between the "average alloy" and the true random alloy. The Helmholtz Free Energy is defined within the NVT ensemble, and we use the volume corresponding to zero pressure in the true random alloy at each desired temperature. The method used here for calculating free energy differences is based on Kirkwood's coupling parameter approach [14], which is similar to thermodynamic integration [15] and the slow growth method [16]. In all of these methods, the free energy difference is obtained using a transformation of the system Hamiltonian $\mathscr{H}$ from the random alloy to the $A$-atom material. Specifically, the Hamiltonian $\mathscr{H}$ is parametrized by a scalar variable $\lambda \in[0,1]$ with $\mathscr{H}(0)$ being the system of interest and $\mathscr{H}(1)$ being the reference system.

Following Skinner et al. [17] and Ravelo et al. [18], we use an "alchemical" transformation where atom types are transformed from the true elemental atoms to the $A$-atom at each atomic site via linear interpolation of the mass and the EAM potential. Specifically, for an atom site that is occupied by a type- $X$ atom in the true random alloy, the mass and potential
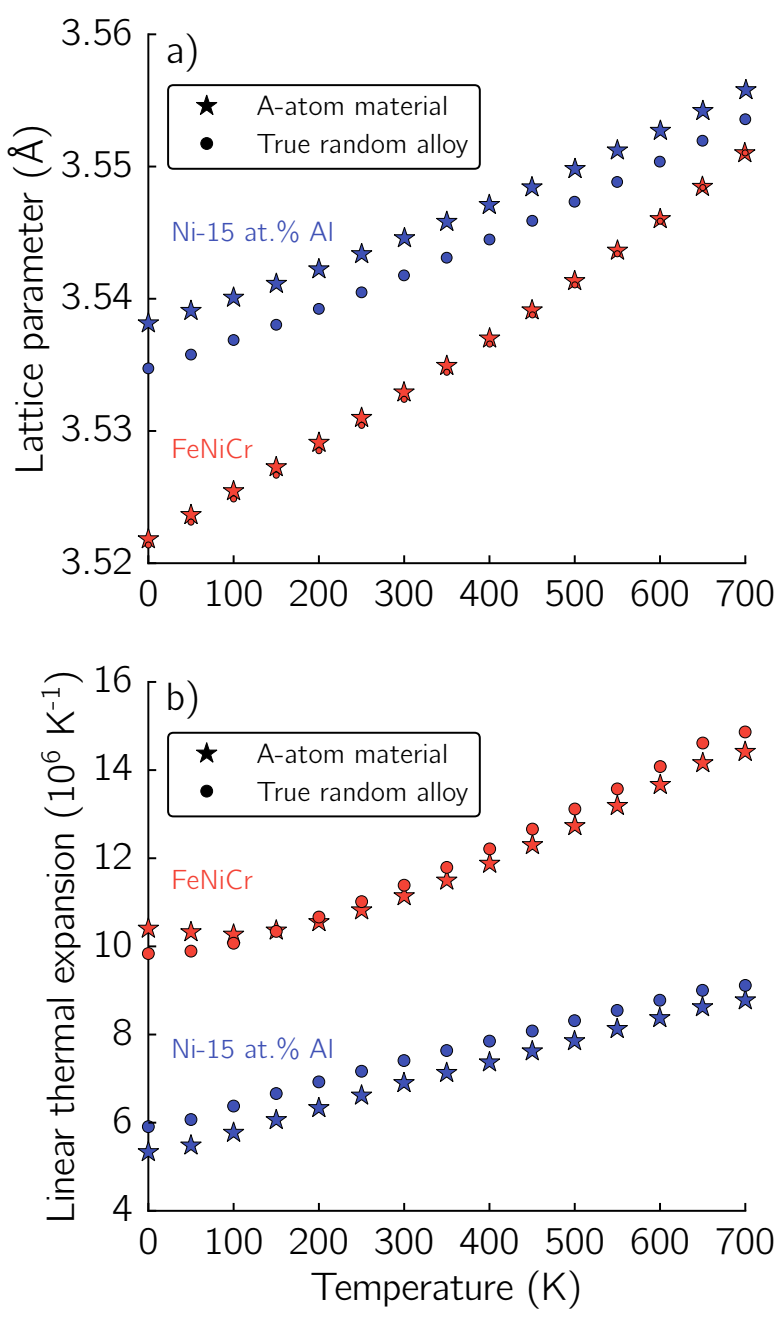

FIG. 1. a) Average FCC lattice parameter versus temperature, for two different alloy systems and for both true random alloys (average of three samples) and the "average atom" material. Circle size spans the maximum variations observed across the entire dataset. b) Linear thermal expansion coefficient versus temperature, for the cases shown in (a).

functions are transformed as

$$
\begin{aligned}
m^{\tilde{X}} & =\lambda m^{A}+(1-\lambda) m^{X}, \\
F^{\tilde{X}} & =\lambda F^{A}+(1-\lambda) F^{X}, \\
\rho^{\tilde{X}} & =\lambda \rho^{A}+(1-\lambda) \rho^{X}, \\
V^{\tilde{X} \tilde{Y}} & =\sum_{X, Y}^{N_{T}+1} \tilde{c}^{X} \tilde{c}^{Y} V^{X Y},
\end{aligned}
$$

where $\tilde{X}$ and $\tilde{Y}$ denote the interpolated types; the summation over atom types now also includes the A-atom type; and $\tilde{c}^{X}$ is $\lambda$ if $X$ is the A-atom and $(1-\lambda)$ if it is a real type. A comparison with Eq. 5 reveals that this interpolation is actually equivalent to defining a new atom at each atomic site that is the average of the $A$-atom and the true atom $\mathrm{X}$ at 
This is an author-created, un-copyedited version of an article accepted for publication/published in Modelling and Simulation in Materials Science and Engineering. IOP Publishing Ltd is not responsible for any errors or omissions in this version of the manuscript or any version derived from it. The Version of Record is available online at http://dx.doi.org/10.1088/0965-0393/24/4/045017. Citation details: Nöhring,

W. G.; Curtin, W. A. Model. Simul. Mater. Sci. Eng. 2016, 24 (4), 45017.

"concentrations" $\lambda$ and $(1-\lambda)$. Rather than implementing the interpolation within the MD code, it is thus more convenient to create all of these new interpolated atom potentials and masses in advance as separate new EAM atom types. Thus, new potential were created for 35 concentrations $\lambda$ $\{0,0.025,0.05\} \cup\{0.07,0.10,0.13, \ldots, 1.0\}[19]$ and simulations at any $\lambda$ are performed simply by calling the corresponding new EAM atom type.

To evaluate the free energy differences, we use the method of de Koning and Antonelli [20,21] based on a thermodynamic analysis that is not specific to any particular thermostat. De Koning and Antonelli considered a system of interest $\mathbb{S}_{1}$ that is coupled to a thermostat $\mathbb{S}_{2}$ and to an external work source $\mathbb{W}$. The system $\mathbb{S}_{1}$ must be in thermal equilibrium with $\mathbb{S}_{2}$ at temperature $T$, while the extended system $\mathbb{S}_{1} \cup \mathbb{S}_{2}$ must be isolated except for the coupling to $\mathbb{W}$ through the variable $\lambda$. As $\lambda$ changes, $\mathbb{S}_{1}$ exchanges reversible work (but no heat) with $\mathbb{W}$. Under these conditions, the free energy of $\mathbb{S}_{1}$ changes by [20]:

$$
\Delta A=\Delta U_{1}+\Delta U_{2},
$$

where $\Delta U_{1}$ and $\Delta U_{2}$ are the changes in internal energy of $\mathbb{S}_{1}$ and $\mathbb{S}_{2}$, respectively.

Applying the general concept to our system, the alloy is $\mathbb{S}_{1}$ and the change of the interatomic potential according to Eq. 7 can be interpreted as the work performed on the atoms by a fictitious $\mathbb{W}$. A Nosé-Hoover chain (NHC) thermostat [2224] serves as $\mathbb{S}_{2}$. With this choice of thermostat, the dynamics preserves the extended Hamiltonian [24]

$$
\begin{aligned}
\mathscr{H}(\vec{p}, \vec{q}, \vec{\zeta}, \vec{\eta}, \lambda) & =E(\vec{q}, \lambda)+\sum_{i}^{N} \sum_{k=1}^{3} \frac{p_{i, k}^{2}}{2 m^{X}(\lambda)} \\
& +\sum_{i}^{N_{C}} \frac{\zeta_{i}^{2}}{2 Q_{i}}+N k_{B} T \eta_{1}+\sum_{i}^{N_{C}} k T \eta_{i},
\end{aligned}
$$

where $N_{C}$ is the number of chain elements, $\vec{p}$ and $\vec{q}$ are the $3 N$ vectors of particle positions and momenta, respectively, $p_{i, k}$ is the momentum of atom $i$ along along direction $k, \vec{\zeta}$ and $\vec{\eta}$ are $N_{C}$-vectors for the dimensionless positions and momentumlike variables of the thermostat chain elements, $E(\vec{q}, \lambda)$ is Eq. 4 with the EAM functions of Eq. 7; $k_{B}$ is Boltzmann's constant, and $Q_{i}$ is the "mass" (units of energy $\times$ time $^{2}$ ) of chain element $i$. The above system is compatible with the requirements of the method, since at fixed $\lambda$, (i) $\mathbb{S}_{1}$ is kept at constant temperature by $\mathbb{S}_{2}$ and (ii) the extended system conserves energy. $\mathbb{W}$ can then exchange work with $\mathbb{S}_{1}$ in a (theoretically) reversible way by changing $\lambda$ and $\mathbb{S}_{2}$ is not related to $\lambda$, and therefore not connected to $\mathbb{W}$. Overall, switching from $\lambda=0$ (the random solid solution) to $\lambda=1$ (the average material) allows for the difference in Helmholtz free energy to be computed.

When applying the above method, there are two practical concerns: reversibility and ergodicity. The exchange of work with $\mathbb{W}$ is reversible only in theory. Due to the finite and abrupt change of $\lambda$ at each increment of $\lambda$ and a finite simulation time, measurements are not performed in full equilibrium and the transformation is not fully reversible. To esti- mate errors due to irreversibility, we have simulated the forward $\lambda=0 \rightarrow 1$ path and then the reverse $\lambda=1 \rightarrow 0$ paths. The irreversible energy change is the difference between the internal energies of the extended system at the end and at the beginning of this cycle. The reversible free energy difference is then estimated by taking the mean value of $\Delta A$ obtained for the forward and reverse paths. Regarding ergodicity, recent numerical tests suggest that NHC thermostats create ergodic systems [25] so that it should be possible to sample the most important energy states within attainable simulation times.

Free energy difference calculations were performed for the same three random solutions with exactly the same distribution of atom types used in the lattice parameter calculations (Sec. III). For each random sample and each temperature $(50 \ldots 700 \mathrm{~K})$, a perfect lattice was generated using the average lattice parameter for each spatial direction. Random velocities corresponding to twice the target temperature were then assigned to the atoms, and the target temperature was stabilized by performing Langevin dynamics for $50 \mathrm{ps}$. Finally, the samples were equilibrated for $200 \mathrm{ps}$ in the canonical $(N V T)$ ensemble using the NHC thermostat with three elements and a temperature damping parameter of $100 \mathrm{ps}$ and timestep of $1 \mathrm{fs}$. At the beginning of the free energy calculation, the sample was kept at the target temperature for another $10 \mathrm{ps}$, in order to start up the NHC thermostat, and to continue equilibration. After this initialization phase, the forward and reverse transformation were simulated. For each $\lambda$, the appropriate EAM potential was applied, the system was equilibrated for $10 \mathrm{ps}$, and the required thermodynamic variables then recorded over $50 \mathrm{ps}$.

\section{B. Results}

In the range $0-700 \mathrm{~K}$, the free energy difference $\Delta A$ between the true random solution and the corresponding $A$-atom material is $0.056-0.063 \mathrm{eV}$ per atom in $\mathrm{FeNiCr}$ and 0.0084$0.0116 \mathrm{eV}$ per atom in $\mathrm{Ni}_{85} \mathrm{Al}_{15}$, see Fig. 2. In both cases, the major portion of the free energy difference is associated with the difference at $T=0 \mathrm{~K}$, which is the difference in cohesive energy plus a mechanical contribution because the homogeneous material is studied at the volume of the true random solution. However, the mechanical contribution is only $1 \%$ of the difference at $T=0 \mathrm{~K}$, so the difference in cohesive energy between random and $A$-atom materials dominates. The absolute cohesive energies of $\mathrm{FeNiCr}$ and $\mathrm{Ni}_{85} \mathrm{Al}_{15}$ are listed in table I, and the differences between random solutions and $A$-atom material are $1.3 \%$ and $0.18 \%$ of the random solution cohesive energy, respectively.

TABLE I. Cohesive energy in eV.

\begin{tabular}{ccc}
\hline Alloy & True random alloy & A-atom material \\
\hline $\mathrm{FeNiCr}$ & 4.2029 & 4.1973 \\
$\mathrm{Ni}_{85} \mathrm{Al}_{15}$ & 4.5277 & 4.5192 \\
\hline
\end{tabular}

The free energy difference $\Delta A$ increases nearly linearly with temperature, thus corresponding to an entropy difference 
This is an author-created, un-copyedited version of an article accepted for publication/published in Modelling and Simulation in Materials Science and Engineering. IOP Publishing Ltd is not responsible for any errors or omissions in this version of the manuscript or any version derived from it. The Version of Record is available online at http://dx.doi.org/10.1088/0965-0393/24/4/045017. Citation details: Nöhring,

W. G.; Curtin, W. A. Model. Simul. Mater. Sci. Eng. 2016, 24 (4), 45017.

$\Delta S=\partial \Delta A / \partial T$ of $\approx 0.010 k_{B}$ per atom for FeNiCr and 0.047 $0.049 k_{B}$ per atom for $\mathrm{Ni}_{85} \mathrm{Al}_{15}$. This is a very small difference in entropy, and is much smaller than the configurational entropy of the random alloy $\left(S_{\text {config }}=-N k_{B} \sum_{X} c^{X} \log \left(c^{X}\right)\right)$ that is not computed in direct simulations of random alloys. As expected, the numerical transformation is not fully reversible. However, the dissipated energy is always at least one order of magnitude lower than $\Delta A$. The free energy differences are thus negligible for most applications of the $A$-atom method, thus validating the method up to temperatures spanning a wide range of interest for understanding mechanical properties of these and similar materials.

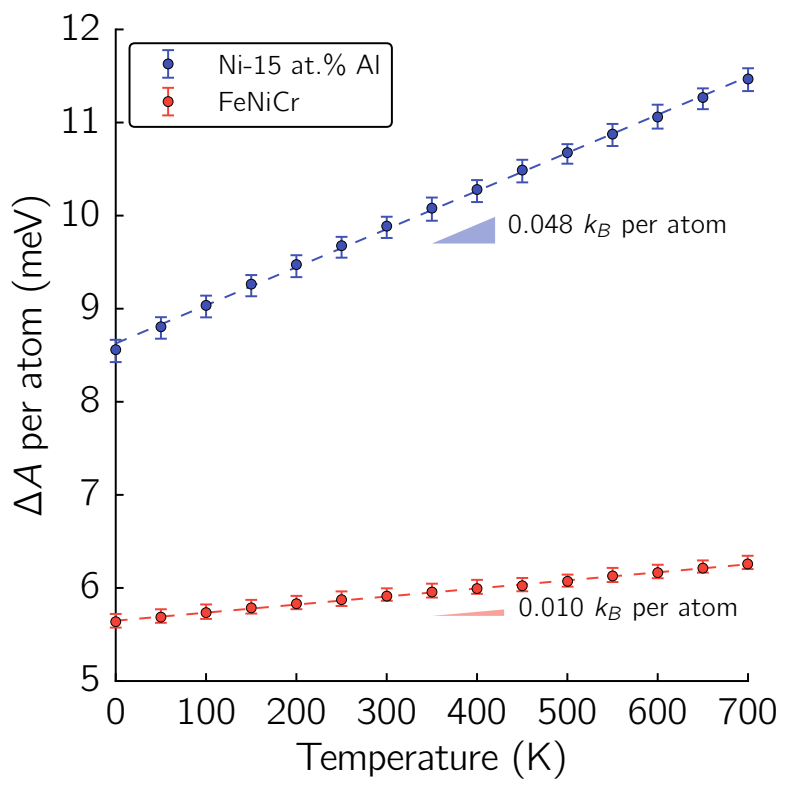

FIG. 2. Free energy difference between the "average atom" material and the corresponding true random alloy versus temperature for $\mathrm{Ni}_{85} \mathrm{Al}_{15}$ (blue symbols) and $\mathrm{FeNiCr}$ (red symbols). For random alloys, each data point is the average over three different random configurations and errorbars indicate the maximum and minimum values. Dashed lines: linear fit revealing the difference in vibrational entropy between "average atom" and true random alloys.

\section{ELASTIC CONSTANTS}

In a linear anisotropic material, the infinitesimal strains $\varepsilon_{m n}$ $(m, n=1,2,3)$ and the stresses $\sigma_{k l}(k, l=1,2,3)$ are related through the components $C_{k l m n}$ of the (fourth-order) tensor of elastic stiffness as

$$
\sigma_{k l}=C_{k l m n} \varepsilon_{m n}
$$

where Einstein's summation convention applies. The averageatom material has cubic symmetry with three independent non-zero values $C_{1111}=C_{2222}=C_{3333}, C_{1122}=C_{1133}=C_{2233}$, and $C_{1212}=C_{1313}=C_{2323}$. The (finite-size) random solid solutions are slightly orthotropic because the distribution of atom types is not exactly the same along the three spatial directions. For this non-cubic case, the degeneracy of the elastic constants is lost and there are nine non-zero elastic constants. We use two methods to compute the $C_{k l m n}$ : stress-strain tests for a few components only and the stress fluctuation method [26].

To compute the $C_{k l m n}$ using stress-strain tests, we apply a desired $\varepsilon_{m n}$ and measure the resulting $\sigma_{k l}$, calculating the corresponding $C_{k l m n}$ from Eq. 10 . To obtain accurate results at finite temperatures $\varepsilon_{m n}$ must be large enough to create $\sigma_{k l}$ that are significantly larger than thermal fluctuations but $\varepsilon_{m n}$ must also be small enough so that the response remains in the linear range. Furthermore, successive increments of deformation create elastic waves that dampen slowly and delay convergence of the average $\sigma_{k l}$ to the correct value. Considering all of these issues, we impose one negative and one positive strain increment of magnitude $10^{-3}$. For $\mathrm{Ni}_{85} \mathrm{Al}_{15}$ and $T=0 \mathrm{~K}$, tests show that the error due to nonlinearity at this strain level is on the order of a few MPa yet with stresses large enough to be accurately determined.

We imposed two deformations, uniaxial strain along the 2 direction, with the response

$$
\begin{aligned}
& \sigma_{22}=C_{2222} \varepsilon_{22}, \\
& \sigma_{11}=C_{1122} \varepsilon_{22}, \\
& \sigma_{33}=C_{2233} \varepsilon_{22}
\end{aligned}
$$

and simple shear $\gamma_{12}$, with the response

$$
\sigma_{12}=C_{1212} \gamma_{12}
$$

We have used the same random alloy samples as used in the free energy calculations, see Sec. IV A. The strain was imposed gradually over a time of $20 \mathrm{ps}$, the samples were then equilibrated for $20 \mathrm{ps}$, and finally the stress was computed as the average stress over an interval of $150 \mathrm{ps.} \mathrm{A} \mathrm{NHC} \mathrm{ther-}$ mostat with three elements was used to control temperature as described earlier.

The fluctuation method determines $C_{k l m n}$ from the fluctuations and ensemble averages given, in the NVE or NVT ensemble, as [26]:

$$
\begin{aligned}
C_{k l m n} & =-\frac{V}{k_{B} T}\left(\left\langle\sigma_{k l} \sigma_{m n}\right\rangle-\left\langle\sigma_{k l}\right\rangle\left\langle\sigma_{m n}\right\rangle\right), \\
& +\frac{2 N k_{B} T}{V}\left(\delta_{k m} \delta_{l n}+\delta_{k n} \delta_{l m}\right), \\
& +\left\langle{ }^{1} B_{k l m n}\right\rangle+\left\langle{ }^{2} B_{k l m n}\right\rangle+\left\langle{ }^{3} B_{k l m n}\right\rangle,
\end{aligned}
$$

where $V$ is the cell volume, $T$ is the temperature, $\delta_{k m}$ is Kronecker's delta, and $\langle\ldots\rangle$ indicates the ensemble average. The Born terms ${ }^{x} B_{k l m n}(x=1,2,3)$ are functions of the first and second derivative of the potential energy [27]. At zero temperature, and in the absence of internal strains, $C_{k l m n}$ is the sum only of these Born terms [28]. We also note that the first term in Eq. 13 contains the stress fluctuations and a factor of $1 / T$. Calculations were performed on undeformed samples identical to those used for the stress-strain tests. After an initial equilibration phase of $10 \mathrm{ps}$, the samples were held at the 
This is an author-created, un-copyedited version of an article accepted for publication/published in Modelling and Simulation in Materials Science and Engineering. IOP Publishing Ltd is not responsible for any errors or omissions in this version of the manuscript or any version derived from it. The Version of Record is available online at http://dx.doi.org/10.1088/0965-0393/24/4/045017. Citation details: Nöhring,

W. G.; Curtin, W. A. Model. Simul. Mater. Sci. Eng. 2016, 24 (4), 45017.

desired constant temperature for 500 ps to calculate the canonical ensemble averages in Eq. 13. The temperature was again controlled by the three-element NHC.

Figs. 3 and 4 show the computed averged elastic constants. Note that simplified Voigt notation is now used $C_{k k k k} \rightarrow C_{11}$, $C_{k k l l} \rightarrow C_{12}$ and $C_{k l k l} \rightarrow C_{44}$ and the differences among nomimally identical $C_{k l m n}$ under cubic symmetry are indeed small $( \pm 0.5 \mathrm{GPa})$ in the true random alloys. For FeNiCr and $T \geq$ $50 \mathrm{~K}$, the differences between the true random alloy and $A$ atom elastic constants are within $\pm 2-3 \%$ of the true random alloy values. For $\mathrm{Ni}_{85} \mathrm{Al}_{15}$, the deviation of $C_{11}$ is comparatively large at low temperature, $5-6 \%$ (ca. $11 \mathrm{GPa}$ ) at $50 \mathrm{~K}$ but with better agreement for $C_{12}$ and $C_{44}$ (less or equal to $2.5 \%$ or ca. $4 \mathrm{GPa}$ and $1.1 \%$ or ca. $1 \mathrm{GPa}$, respectively).
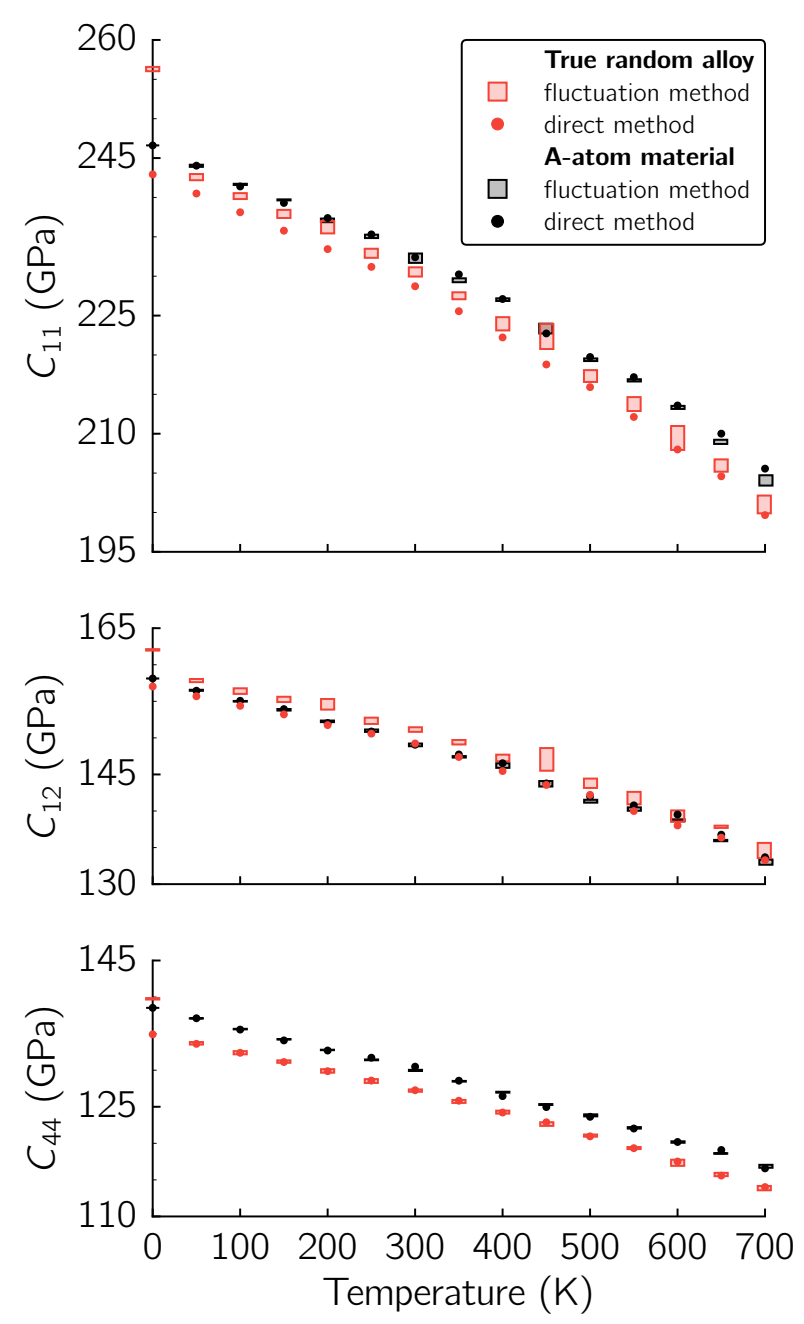

FIG. 3. Average cubic elastic constants of FeNiCr versus temperature, as computed by different methods for the true random alloy and "average atom" material using Voigt notation. Symbol size for the fluctuation method indicates the minimum and maximum values among all (cubic) symmetry-equivalent values across the three random samples. Symbols for the direct method show the average value, with variations smaller than those obtained using the fluctuation method.
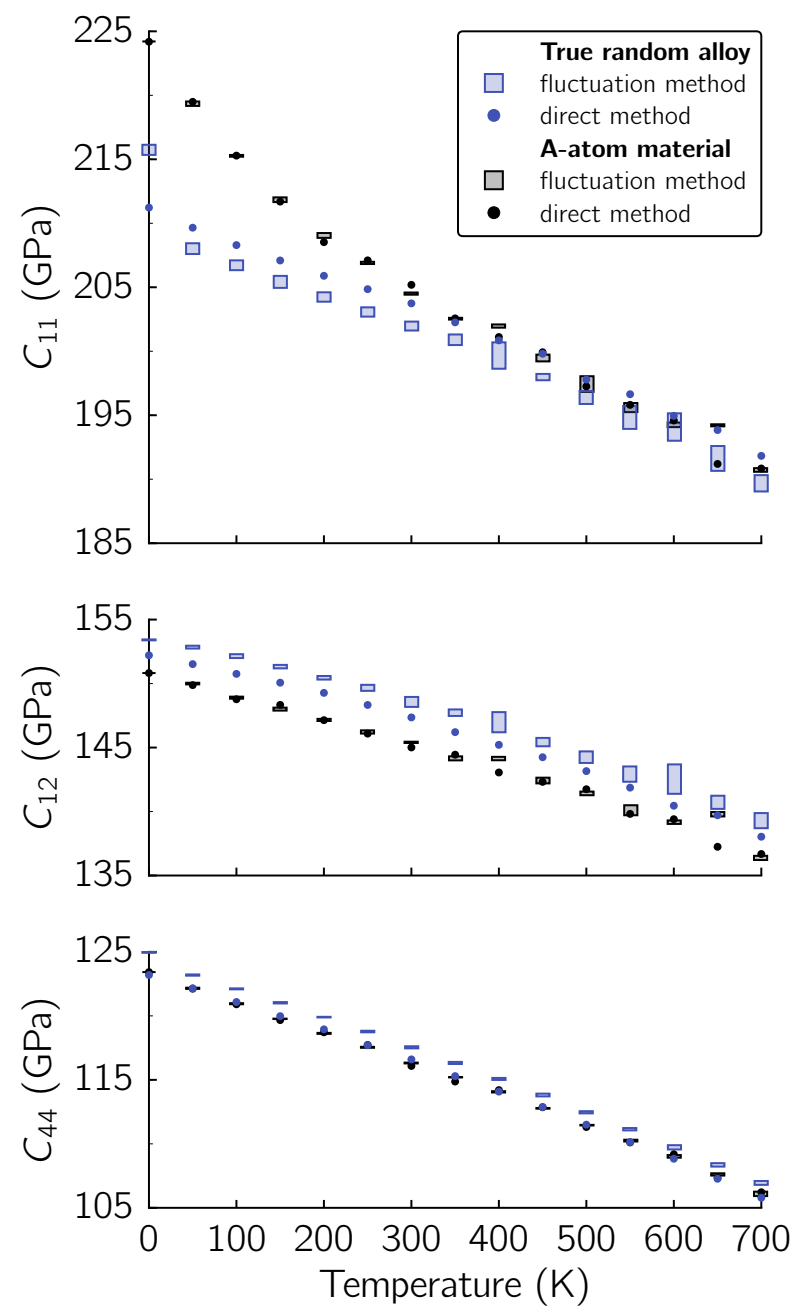

FIG. 4. Average cubic elastic constants of $\mathrm{Ni}_{85} \mathrm{Al}_{15}$ versus temperature, as computed by different methods for the true random alloy and "average atom" material using Voigt notation. Symbol size for the fluctuation method indicates the minimum and maximum values among all (cubic) symmetry-equivalent values across the three random samples. Symbols for the direct method show the average value, with variations smaller than those obtained using the fluctuation method.

Besides the difference between the true random alloys and the $A$-atom material, there are differences between methods even for the same material. These differences can be considered as a measure of the uncertainty/accuracy of elastic moduli even when computed with high attention to detail as done here. The differences between true random alloys and the $A$ atom material exceed the differences due to method alone, indicating real (but still small) errors associated with the $A$-atom method.

For the true random alloy, there is an important deviation between the two methods at $0 \mathrm{~K}$. Results using the fluctuation method for the true random alloy elastic constants jump by a few GPa relative to the values at $T=50 \mathrm{~K}$ for all cases of $\mathrm{FeNiCr}$, and for $C_{11}$ of $\mathrm{Ni}_{85} \mathrm{Al}_{15}$. Additional calculations for 
This is an author-created, un-copyedited version of an article accepted for publication/published in Modelling and Simulation in Materials Science and Engineering. IOP Publishing Ltd is not responsible for any errors or omissions in this version of the manuscript or any version derived from it. The Version of Record is available online at http://dx.doi.org/10.1088/0965-0393/24/4/045017. Citation details: Nöhring,

W. G.; Curtin, W. A. Model. Simul. Mater. Sci. Eng. 2016, 24 (4), 45017.

one random solution in the range $5-50 \mathrm{~K}$ indicate that there is a jump at $T=0 \mathrm{~K}$, not simply a rapid but continous variation. This jump is an artifact of the fluctuation method. In the fluctuation method, only the Born terms are evaluated at $0 \mathrm{~K}$ and a jump can occur if the fluctuation contribution does not go to zero as $T \rightarrow 0 \mathrm{~K}$ [28]. Indeed, for $\mathrm{Ni}_{85} \mathrm{Al}_{15}$, the fluctuation terms tends to zero for the $A$-atom material and no jump is observed (Fig. 5) whereas the fluctuation contribution to $C_{11}$ in the true random alloy tends towards a nonzero value (ca. $-6 \mathrm{GPa}$ ) consistent with the jump of $C_{11}$ in Fig. 4. According to Ray [26], the problem arises for systems with initial internal strains, which is precisely the situation in the true random alloys. In contrast, the $A$-atom material has a perfect fcc lattice with no internal strains and hence the elastic constants are the sums of only the Born terms, and no jumps are observed. Thus, at $T=0 \mathrm{~K}$, the fluctuation method should not be used in random alloys.

Finally, we note that the differences among methods and systems depends on how well the ensemble averages have converged. For $\mathrm{Ni}_{85} \mathrm{Al}_{15}$ at $T=0 \mathrm{~K}$, all $C_{k l m n}$ have converged to within fractions of a GPa within our simulation time. However, at $T=700 \mathrm{~K}$, only $C_{k l k l}$ has converged comparably, while $C_{k k k k}$ and $C_{k k l l}$ are still varying by 1-2 GPa. However, the difference between the true random alloy and the $A$-atom data has converged to less than one GPa. Evaluating the individual contributions in Eq. 13, we find that the worse convergence of $C_{k k k k}$ and $C_{k k l l}$ relative to $C_{k l k l}$ is caused by the stress fluctuation term. The slow convergence of this term, in turn, is a consequence of slow (half-period $500 \mathrm{ps}$ ) oscillations of $\sigma_{11}, \sigma_{22}$, and $\sigma_{33}$, on the order of $10 \mathrm{MPa}$.

\section{DISCUSSION AND SUMMARY}

The $A$-atom interatomic potential has been shown to predict finite-temperture properties and Helmholtz Free Energies in very good agreement with the true random alloy of the same composition. Lattice parameters agree to within fractions of a percent, while the derivative properties of thermal expansion and elastic constants agree to within a few percent over the entire temperature range. Such agreement is generally quite acceptable, particularly considering that EAM potentials typically have a mismatch of a few percent versus experimentally measured values. At the mechanistic level, the differences in elastic constant will lead to small differences in stored mechanical energy that is available to drive mechanical phenomena, but on the scale of important energies these differences are probably not significant. For phenomena having well-established theoretical foundations, the effects of elastic modulus differences can be assessed quantitatively as needed. The Helmholtz Free Energy differences are also very small, with a primary difference in the cohesive energy at $T=0 \mathrm{~K}$ and a very small difference in entropy on the order of 0.01 $0.05 k_{B}$ per atom. In these respects, the $A$-atom potential thus provides an excellent representation of the corresponding true random alloy thermodynamics.

The analysis of Wojtowicz and Kirkwood [7] showed that the lattice dynamics of a virtual crystal (nearly equivalent to

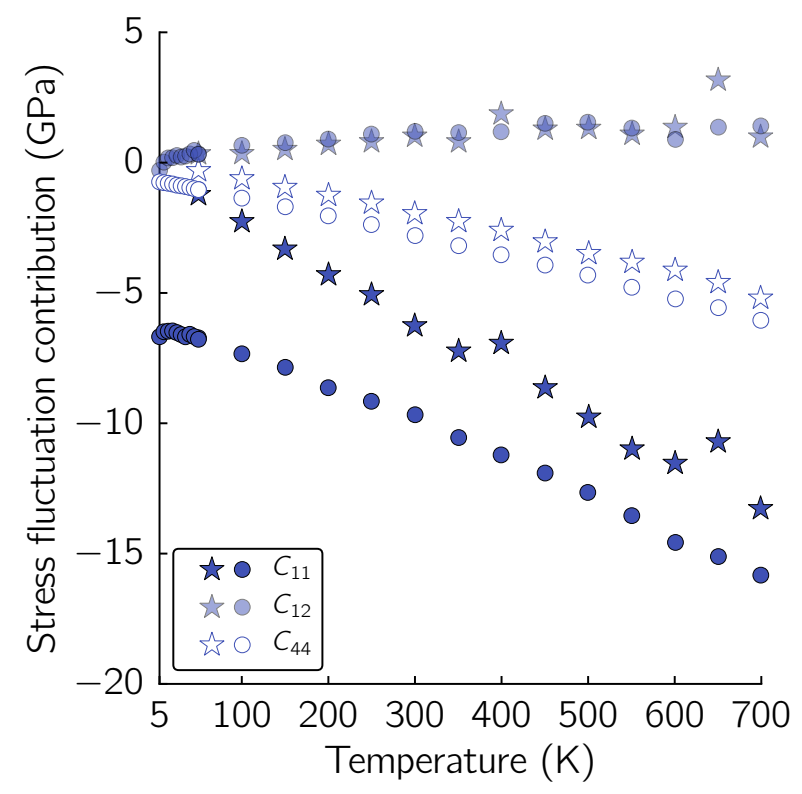

FIG. 5. Stress fluctuation contribution (first term on the right hand side of Eq. 13) to the elastic constants versus temperature, for $\mathrm{Ni}_{85} \mathrm{Al}_{15}$ true random alloys (circles) and "average-atom" materials (stars). The contribution to $C_{11}$ for the true random alloy tends towards a nonzero value for $T \rightarrow 0 \mathrm{~K}$, consistent with the jump in the computed $C_{11}$ at $0 \mathrm{~K}$, see Fig. 4.

our "average atom" system) is the same, to first order, of a truly random alloy. Our results show that the neglected higherorder terms are quite small, and our results go beyond the harmonic approximation. Otherwise, the similarity between the virtual crystal and the average atom approach suggests that phonon-controlled material parameters such as the thermal expansion and elastic constants should be similar. We note also, however, that the analysis of Wojtowicz and Kirkwood assumed that the constituent atom types have approximately equal mass whereas our good results for $\mathrm{Ni}_{85} \mathrm{Al}_{15}$ are in a system with a factor of 2 difference in the atomic masses ( $m=58.71 \mathrm{u}$ for Ni vs. $m=26.98 \mathrm{u}$ for $\mathrm{Al}$ ).

We believe that the $A$-atom potential is particularly useful when combined with the original elemental atoms, for calculating interactions energies between the elemental "solutes" and defects in the average material. The present results show that there is a small mismatch between the $A$-atom material and the true random alloy, which will be manifested in small errors for the interactions energies. That is, the elemental solutes in the random alloy would interact with defects in a matrix that does not quite match the actual average properties of the random alloy matrix. The major differences would occur at zero temperature, however, and the results here indicate that additional entropic contributions to such interaction energies should be quite small. Similar considerations apply for applications where the $A$-atom is used for a (solid solution) matrix containing intermetallic precipitates, with the latter represented explicitly by the component atoms (e.g. $\mathrm{Ni}_{3} \mathrm{Al}$ in a 
This is an author-created, un-copyedited version of an article accepted for publication/published in Modelling and Simulation in Materials Science and Engineering. IOP Publishing Ltd is not responsible for any errors or omissions in this version of the manuscript or any version derived from it. The Version of Record is available online at http://dx.doi.org/10.1088/0965-0393/24/4/045017. Citation details: Nöhring, W. G.; Curtin, W. A. Model. Simul. Mater. Sci. Eng. 2016, 24 (4), 45017.

$\mathrm{Ni}$-Al solid solution matrix). Differences in lattice parameter, thermal expansion, and elastic constants when using the $A$-atom description of the matrix material would lead to small differences in mismatch stresses, with similar small effects on consquent plasticity and other deformation phenomena. However, since the original atom EAM potentials do not exactly correspond with experimental systems, the differences found upon using the $A$-atom potential are likely small compared to differences with real experiments. Finally, the $A$-atom potential might be useful for thermodynamic calculations of phase behavior involving random solution phases, but in this case small difference in free energy may not be negligible. For instance, the order-disorder free energy for $\mathrm{Ni}_{3} \mathrm{Al}$ has been calculated by Ravelo et al. [18] as $0.089-0.083 \mathrm{eV}$ per atom in the range $200-700 \mathrm{~K}$. The free energy difference between the true solid solution and the "average atom" solid solution that we compute here is $\Delta A \sim 11-14 \%$ of the above value. This difference is not too large, and is much smaller than the configurational entropy difference, but might have small consequences for predicted phase behavior.

In summary, the $A$-atom EAM potential derived from a formal averaging of the EAM potentials of true individual atoms in a random alloy has been shown to have negligible deviations from the true random alloy for a number of thermomechanical properties over a wide temperature range, as demonstrated in two different model alloy systems. The general method is not limited in the number or type of components, and thus should find valuable applications in a range of problems in alloy metallurgy. In future publications, we will report on applications of the method to High Entropy Alloys [10], to cross-slip in solid solution alloys, and to crack tip dislocation emission.
[1] R. W. Smith and G. S. Was, Physical Review B 40, 10332 (1989).

[2] R. Najafabadi, H. Wang, D. J. Srolovitz, and R. LeSar, Acta Metallurgica et Materialia 39, 3071 (1991).

[3] C. Varvenne, A. Luque Gomez, W. Nöhring, and W. Curtin, Submitted (2015).

[4] M. S. Daw and M. Baskes, Physical Review B 29, 6443 (1984).

[5] L. Nordheim, Annalen der Physik 401, 607 (1931).

[6] T. Muto, Scientific Papers of the Institute of Physical and Chemical Research 34, 377 (1938).

[7] P. J. Wojtowicz and J. G. Kirkwood, The Journal of Chemical Physics 33, 1299 (1960).

[8] G. Bonny, D. Terentyev, R. C. Pasianot, S. Poncé, and A. Bakaev, Modelling and Simulation in Materials Science and Engineering 19, 085008 (2011).

[9] G. P. Purja Pun, V. Yamakov, and Y. Mishin, Modelling and Simulation in Materials Science and Engineering 23, 065006 (2015).

[10] J.-W. Yeh, S.-K. Chen, S.-J. Lin, J.-Y. Gan, T.-S. Chin, T.-T. Shun, C.-H. Tsau, and S.-Y. Chang, Advanced Engineering Materials 6, 299 (2004).

[11] S. Plimpton, Journal of Computational Physics 117, 1 (1995).

[12] T. Schneider and E. Stoll, Physical Review B 17, 1302 (1978).

[13] H. J. C. Berendsen, J. P. M. Postma, W. F. van Gunsteren, A. DiNola, and J. R. Haak, The Journal of Chemical Physics 81, 3684 (1984).

[14] J. G. Kirkwood, The Journal of Chemical Physics 3, 300 (1935).

[15] J. Q. Broughton and G. H. Gilmer, The Journal of Chemical Physics 79, 5095 (1983).

[16] T. P. Straatsma, H. J. C. Berendsen, and J. P. M. Postma, The Journal of Chemical Physics 85, 6720 (1986).

[17] A. J. Skinner, J. V. Lilli, and J. Q. Broughton, Modelling and
Simulation in Materials Science and Engineering 3, 359 (1995).

[18] R. Ravelo, J. Aguilar, M. Baskes, J. E. Angelo, B. Fultz, and B. L. Holian, Physical Review B 57, 862 (1998).

[19] The slight refinement near $\lambda=0$ was done because preliminary simulations had indicated that the internal energy changes more rapidly with $\lambda$ in this region.

[20] M. de Koning and A. Antonelli, Physical Review E 53, 465 (1996).

[21] M. de Koning and A. Antonelli, Physical Review B 55, 735 (1997).

[22] S. Nosé, Molecular Physics 52, 255 (1984).

[23] W. G. Hoover, Physical Review A 31, 1695 (1985).

[24] G. J. Martyna, M. L. Klein, and M. Tuckerman, The Journal of Chemical Physics 97, 2635 (1992).

[25] P. K. Patra, J. C. Sprott, W. G. Hoover, and C. G. Hoover, Molecular Physics , 1 (2015).

[26] J. R. Ray and A. Rahman, The Journal of Chemical Physics 80, 4423 (1984).

[27] R. J. Wolf, K. A. Mansour, M. W. Lee, and J. R. Ray, Physical Review B 46, 8027 (1992).

[28] J. R. Ray, Computer Physics Reports 8, 109 (1988).

\section{ACKNOWLEDGMENTS}

Support for this work was provided by the European Research Council through the Advanced Grant "Predictive Computational Metallurgy", ERC Grant agreement No. 339081 PreCoMet. WGN thanks Dr. C. Varvenne for helpful discussions during the course of this work. 